\title{
The small RNA SgrS: roles in metabolism and pathogenesis of enteric bacteria
}

\author{
Maksym Bobrovskyy and Carin K. Vanderpool* \\ Department of Microbiology, University of Illinois at Urbana-Champaign, Urbana, IL, USA
}

\section{Edited by:}

Wyndham W. Lathem, Northwestern University Feinberg

School of Medicine, USA

\section{Reviewed by:}

Kai Papenfort, Princeton University, USA

Eric Masse, University of

Sherbrooke, Canada

*Correspondence:

Carin K. Vanderpool, Department of Microbiology, University of Illinois at Urbana-Champaign, C213 CLSL, MC-110, 601 S. Goodwin Ave.,

Urbana, IL 61801, USA

e-mail: cvanderp@life.illinois.edu
Bacteria adapt to ever-changing habitats through specific responses to internal and external stimuli that result in changes in gene regulation and metabolism. One internal metabolic cue affecting such changes in Escherichia coli and related enteric species is cytoplasmic accumulation of phosphorylated sugars such as glucose-6-phosphate or the non-metabolizable analog $\alpha$-methylglucoside-6-phosphate. This "glucose-phosphate stress" triggers a dedicated stress response in $\gamma$-proteobacteria including several enteric pathogens. The major effector of this stress response is a small RNA (sRNA), SgrS. In E. coli and Salmonella, SgrS regulates numerous mRNA targets via base pairing interactions that result in alterations in mRNA translation and stability. Regulation of target mRNAs allows cells to reduce import of additional sugars and increase sugar efflux. SgrS is an unusual sRNA in that it also encodes a small protein, SgrT, which inhibits activity of the major glucose transporter. The two functions of SgrS, base pairing and production of SgrT, reduce accumulation of phosphorylated sugars and thereby relieve stress and promote growth. Examination of SgrS homologs in many enteric species suggests that SgrS has evolved to regulate distinct targets in different organisms. For example, in Salmonella, SgrS base pairs with sopD mRNA and represses production of the encoded effector protein, suggesting that SgrS may have a specific role in the pathogenesis of some $\gamma$-proteobacteria. In this review, we outline molecular mechanisms involved in SgrS regulation of its target mRNAs. We also discuss the response to glucose-phosphate stress in terms of its impact on metabolism, growth physiology, and pathogenesis.

Keywords: small RNA, SgrR, PtsG, glucose-phosphate stress, glycolysis, phosphoenolpyruvate phosphotransferase system
Over the last decade, small RNAs have emerged from relative obscurity to take their places as central regulators of gene expression in organisms from all three domains of life. While hundreds of small RNAs in dozens of bacterial genomes have been identified by computational or experimental methods, the functions of the vast majority of these remain a mystery. Nevertheless, we have learned a great deal about a small number of "model" bacterial sRNAs, including how their production is regulated, what targets they in turn regulate and the physiological outcomes of sRNA-mediated regulation. In this review, we first provide a brief overview of features of regulatory sRNAs that act on mRNAs through base pairing interactions. We then focus on one wellcharacterized sRNA, SgrS (sugar transport related sRNA) and describe its activities on target mRNAs and how these activities regulate bacterial metabolism and pathogenesis.

\section{MECHANISMS OF REGULATION BY BACTERIAL SMALL RNAs}

\section{BASIC CHARACTERISTICS OF SRNA-MEDIATED REGULATION}

Several mechanistic classes of sRNAs have been identified in diverse bacterial species. Many characterized sRNAs act by base pairing with mRNA targets to control mRNA stability and translation. Such sRNAs are often between 50 and 300 nucleotides in length and require an RNA chaperone, Hfq, for their stability and regulatory effects on target mRNAs (Sledjeski et al., 2001; Moller et al., 2002; Zhang et al., 2002). Hff is a hexameric ring protein with sRNA- and mRNA-binding faces (Mikulecky et al., 2004; Link et al., 2009; Zhang et al., 2013). On sRNAs, Hfq binds to stem-loop terminator structures preceded by $\mathrm{A} / \mathrm{U}$ rich sequences (Valentin-Hansen et al., 2004; Otaka et al., 2011; Ishikawa et al., 2012). Hfq-binding sites are located in $5^{\prime}$ untranslated regions (UTRs) of many mRNAs that are regulated by sRNAs (Soper and Woodson, 2008; Link et al., 2009; Salim and Feig, 2010; Salim et al., 2012). Binding of Hfq to both sRNAs and mRNAs increases their local concentrations, stimulates structural remodeling to facilitate pairing and increases annealing rates of cognate pairs (Fender et al., 2010; Maki et al., 2010; Soper et al., 2010; Hopkins et al., 2011). Other factors involved in sRNA-mRNA regulatory transactions include RNase $\mathrm{E}$ and components of the degradosome (Masse and Gottesman, 2002; Masse et al., 2003; Morita et al., 2005). Polynucleotide phosphorylase (PNPase, a $3^{\prime}-5^{\prime}$ exonuclease), RhlB helicase and enolase assemble on RNase $\mathrm{E}$ to form a degradosome complex required for bulk mRNA turnover in proteobacteria (Carpousis, 2007). RNase E binds A/U-rich single-stranded regions of RNAs and is responsible for sRNA-mediated mRNA degradation (Carpousis et al., 2009; Belasco, 2010; Prevost et al., 2011). 


\section{REPRESSION BY SRNAs}

The canonical mechanism of negative regulation by sRNAs involves base pairing interactions that directly inhibit translation initiation because the sRNA sequesters mRNA sequences required for stable ribosome binding, in the region from $\sim 20 \mathrm{nt}$ upstream to $20 \mathrm{nt}$ downstream of the start codon (Beyer et al., 1994; Huttenhofer and Noller, 1994). As more detailed studies of sRNA regulatory mechanisms have been performed, variations on this theme have been discovered. Binding of sRNAs to mRNA sequences outside the region recognized by the ribosome can still inhibit translation initiation (Darfeuille et al., 2007; Sharma et al., 2007; Bouvier et al., 2008; Holmqvist et al., 2010), for example, by recruiting $\mathrm{Hfq}$ to bind at a site overlapping the ribosome binding site (RBS) (Desnoyers and Masse, 2012) or by sequestering mRNA sequences that apparently act as translational enhancer elements (Bandyra et al., 2012; Desnoyers et al., 2013).

Regardless of the mechanism, sRNA-mediated translational repression is often coupled to mRNA degradation by an RNase E degradosome-dependent pathway. Translating ribosomes protect mRNA from RNase E degradation (Dreyfus, 2009), thus sRNA inhibition of translation unmasks RNase $\mathrm{E}$ recognition sites and makes target mRNAs susceptible to degradation (Prevost et al., 2011). Though translational repression and mRNA degradation are typically coupled, this is usually not obligatory for gene silencing. In other words, mutations that abrogate RNase E-dependent turnover of sRNA targets have no impact on translational repression (Morita et al., 2006; Rice and Vanderpool, 2011; Rice et al., 2012). While less commonly described, in some cases sRNA-mediated translational repression has no significant effect on mRNA turnover (Moller et al., 2002). Conversely, there are a handful of known cases where sRNAs do not directly affect translation but rather specifically target mRNAs for degradation (Desnoyers et al., 2009; Pfeiffer et al., 2009).

\section{ACTIVATION BY sRNAs}

sRNAs can also activate gene expression post-transcriptionally. Positive regulation of target mRNAs is achieved via activation of translation or stabilization of the target transcript. Some mRNAs have intrinsic secondary structures in their $5^{\prime}$ UTRs that hinder translation, for example, because the RBS is sequestered. Binding of sRNAs to these $5^{\prime}$ UTRs can prevent formation of translation-inhibitory secondary structures and therefore activate translation (Morfeldt et al., 1995; Lease et al., 1998; Prevost et al., 2007). Another mechanism of activation by sRNAs involves sRNA-mRNA base pairing that alters accessibility of RNase E recognition sites on mRNA targets. sRNA-mRNA binding can induce cleavage of a target transcript, resulting in a processed mRNA with an accessible RBS (Obana et al., 2010) or alternatively sRNA base pairing with an intrinsically unstable mRNA can occlude an RNase E recognition site and prevent cleavage (Papenfort et al., 2013).

\section{THE SRNA SgrS MEDIATES THE RESPONSE TO GLUCOSE-PHOSPHATE STRESS CHARACTERISTICS OF SgrS}

SgrS is a 227-nt (in E. coli) Hfq-binding sRNA (Zhang et al., 2003 ) that is produced during "glucose-phosphate stress," which is characterized by accumulation of phosphosugars like glucose6-phosphate (G6P) or its analog $\alpha$-methyl glucoside-6-phosphate ( $\alpha$ MG6P) (Vanderpool and Gottesman, 2004; Wadler and Vanderpool, 2007). Glucose and $\alpha$-methyl glucoside ( $\alpha \mathrm{MG})$ are taken up and phosphorylated by the phosphoenolpyruvate phosphotransferase system (PTS) (Postma et al., 1993; Deutscher et al., 2006; Gorke and Stulke, 2008). If metabolism of G6P is blocked (e.g., by mutation of genes encoding early glycolytic enzymes), or if cells accumulate non-metabolizable $\alpha$ MG6P, sgrS is induced by the transcription factor SgrR (Figure 1) (Vanderpool and Gottesman, 2007). Both SgrS and SgrR are essential for cell growth under glucose-phosphate stress conditions (Vanderpool and Gottesman, 2004). SgrS regulates a number of mRNA targets through base pairing interactions involving a conserved region near the $3^{\prime}$ end (Figure 2A, conserved residues are in red in Figure 2B). In addition, the $5^{\prime}$ end encodes a 43 -amino-acid protein called SgrT (Figure 2A). Remarkably, SgrS base pairing activity and SgrT function by independent regulatory mechanisms to allow cells to cope with glucose-phosphate stress and continue growing (Figure 1) (Wadler and Vanderpool, 2007; Balasubramanian and Vanderpool, 2013).

\section{TARGETS OF SgrS REGULATION}

EIICB $^{\text {glc }}$, encoded by $p t s G$, mediates transport and phosphorylation of glucose and $\alpha \mathrm{MG}$. SgrS negatively regulates $p t s G$ by directly inhibiting $p t s G$ translation initiation by base pairing with the ptsG 5' UTR near the RBS (Figures 1, 2C). SgrS-dependent translational repression requires $\mathrm{Hfq}$ and stimulates $p t s G \mathrm{mRNA}$ degradation by an RNase E-dependent pathway (Vanderpool and Gottesman, 2004; Kawamoto et al., 2006; Maki et al., 2008). SgrS is highly unstable in the $h f q$ mutant strain, highlighting the essential role of $\mathrm{Hfq}$ in SgrS-dependent regulation (Balasubramanian and Vanderpool, 2013).

SgrS also represses manXYZ, which encodes a PTS transporter of relatively broad substrate specificity. SgrS repression of manXYZ is carried out through a more complex mechanism compared to $p t s G$. Two distinct SgrS binding sites on manXYZ mRNA are located within early manX coding sequences and within the manX-man $Y$ intergenic region (Figures 1, 2C). SgrS binding at the $\operatorname{man} X$ site is responsible for translational repression of $\operatorname{man} X$, but has no effect on translation of $\operatorname{man} Y$ or $\operatorname{man} Z$ (Rice et al., 2012). The intergenic SgrS binding inhibits translation of manY and man $Z$ (translation of man $Y$ and man $Z$ is coupled) and this is independent of manX regulation (Rice et al., 2012). SgrS binding at each site individually does not affect manXYZ mRNA stability; pairing at both sites is required for RNase E-dependent degradation of manXYZ mRNA (Rice et al., 2012).

A third SgrS target, yigL mRNA, which encodes a haloacid dehalogenase-like phosphatase (Koonin and Tatusov, 1994), is positively regulated by SgrS (Papenfort et al., 2013). Synthesis of YigL is induced by SgrS in response to glucose-phosphate stress. The $y i g L$ gene is in an operon with the upstream pldB, however SgrS activates only yigL. In the absence of SgrS, RNase E processing of the pldB-yigL transcript yields an mRNA that is susceptible to further degradation. When SgrS is produced, it base pairs with a sequence on the processed 'pldB-yigL mRNA (Figures 1, 2C) and prevents further degradation. This mechanism of positive 

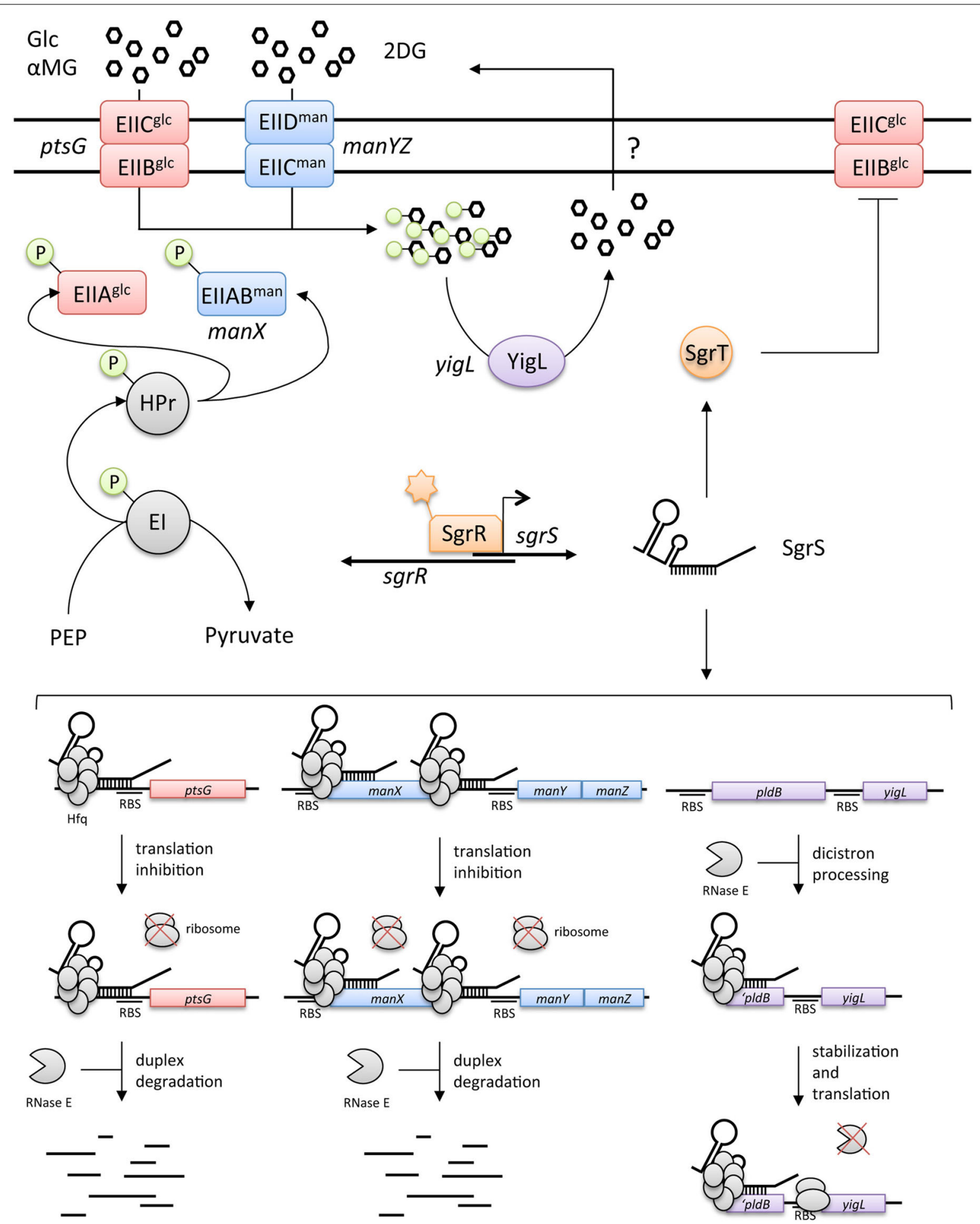

FIGURE 1 | Current model for the role of SgrS in the glucose-phosphate stress response. The top panel illustrates the main features of the phosphoenolpyruvate phosphotransferase system (PTS), which transports numerous carbohydrates as well as glucose analogs ( $\alpha M G, 2 D G$ : $\alpha$-methyl glucoside and 2-deoxy glucose, respectively). Glucose-phosphate stress is associated with accumulation of sugar-phosphates (hexagons with attached green circles). The stress response is initiated by the activated transcription factor, SgrR, which induces SgrS synthesis. SgrS has two functions; the first is base pairing-dependent regulation of target mRNAs (illustrated in lower panel), the second is production of the $\sim 40$ amino acid protein SgrT. SgrT acts to repress activity of the EllCBglc (PtsG) transporter (top panel). The base pairing activity results in repression of two mRNA targets encoding PTS sugar transporters, pts $G$ and manXYZ, and activation of a third mRNA target encoding a phosphatase, yigL (described in detail in the text). Altogether, the base pairing activity of SgrS on these various targets inhibits further uptake of sugar-phosphates by inhibiting production of sugar transporters and promotes sugar efflux by providing neutral sugar substrates that are pumped out by an unknown efflux pump (indicated by a "?"). 
A

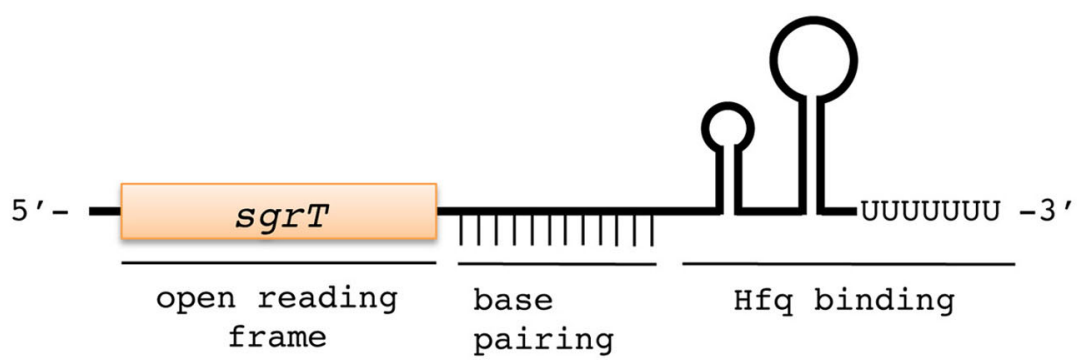

B

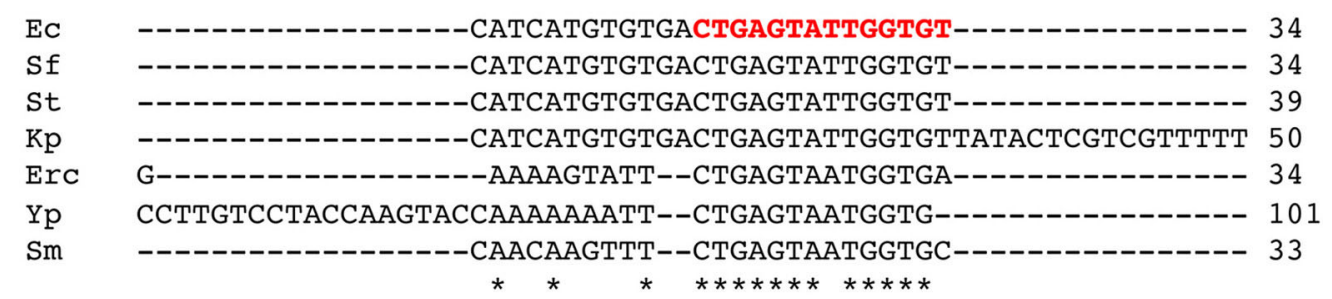

C

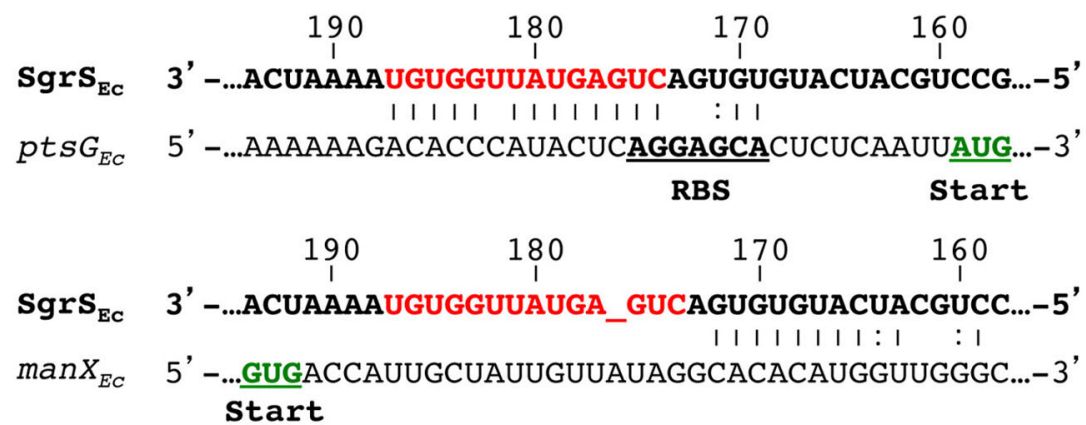
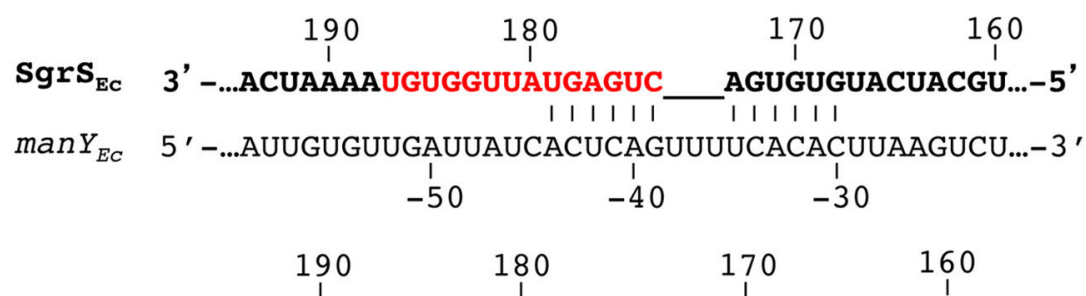

$\operatorname{SgrS}_{\text {St }} \quad 3^{\prime}$-...UAGCGGAUGUGGUUA_UGAGUCAGUGUGUACUACAGACG...-5 '

। ।: । । - : । । । । । । ।: ।: ।

$p l d B_{S t} \quad 5^{\prime}-\ldots$...AAAAGGACGCCAUGCGCUCAGUCGCGCUUAACGCCAUU...-3' $930 \quad 940 \quad 950 \quad 960$

$\begin{array}{llll}190 & 180 & 170 & 160\end{array}$

SgrS $_{\text {St }} \quad 3^{\prime}$-...UAGCGGAUGUGGUUAUGAGUCAGUGUGUACUACAGACG...-5'

$s o p D_{S t} \quad 5^{\prime}-\ldots$...GAAGGAAAAUAUUAUGC_CAGUCACUUUAAGCUUCGgU...- 3 '

$\begin{array}{cc}1 & 1 \\ 1 & 10\end{array}$

FIGURE 2 | Characteristics of SgrS and SgrS-mRNA base pairing interactions. (A) The main functional domains of SgrS are illustrated. The sgrT open reading frame is located at the $5^{\prime}$ end, the conserved base pairing region is downstream of $s g r T$ and upstream of the intrinsic terminator hairpin (which comprises the Hfq-binding domain). (B) Alignment of the base pairing region of SgrS homologs from enteric species. The most conserved region is indicated by asterisks below the alignment and in red for the E. coli (Ec) homolog. Abbreviations for other species: Sf, Shigella flexneri; St, Salmonella enterica serovar Typhimurium; Kp, Klebsiella pneumoniae; Erc, Erwinia carotovora; Yp, Yersinia pestis; Sm, Serratia marcescens. (C) SgrS-mRNA base pairing interactions. Interactions with each confirmed SgrS target are shown (species abbreviations are as in $\mathbf{B}$ ). Watson-Crick base interactions $\mathrm{G}-\mathrm{C}$ and $\mathrm{A}-\mathrm{U}$ are indicated with vertical lines and non-canonical G-U pairs are denoted with two dots. The conserved SgrS base pairing region is indicated in red. Start codons are indicated in green and underlined. Ribosome binding sites (RBS) are bold and underlined. 
regulation is unusual in that an initial processing event is required to allow SgrS access to its binding site because cleavage within $p l d B$ frees the $p l d B$ region from translating ribosomes (Papenfort et al., 2013). Moreover, activation of $y$ igL by SgrS is translationindependent. SgrS stabilizes yigL mRNA by occluding a specific RNase E cleavage site upstream of the yigL coding region, not by enhancing yigL translation (Papenfort et al., 2013). A similar translation-independent mechanism of mRNA stabilization was recently described for the RydC sRNA-cfa mRNA regulatory pair (Frohlich et al., 2013).

\section{GLUCOSE-PHOSPHATE STRESS PHYSIOLOGY}

Targets of SgrS include sugar transporters and a sugar phosphatase. SgrS-mediated repression of sugar transporters diminishes cells' capacity to take up sugars and therefore reduces further phosphosugar accumulation (Figure 1). However, this effect is not immediate: PtsG protein has a half-life of $\sim 80 \mathrm{~min}$ (Papenfort et al., 2013), so merely stopping new synthesis of PtsG would not provide a fast remedy for the problem of phosphosugar accumulation. The activation of YigL synthesis by SgrS addresses this problem since dephosphorylation of sugars allows their efflux (Figure 1) (Winkler, 1971; Haguenauer and Kepes, 1972; Papenfort et al., 2013). Growth competition experiments between wild-type and sgrS mutants provided insight into how regulation of different SgrS targets contributes to stress resistance and growth during glucosephosphate stress (Sun and Vanderpool, 2013). When cells are stressed while growing in rich medium, SgrS-mediated regulation of $p t s G$ mRNA alone is sufficient to confer wild-type levels of growth. In contrast, cells stressed in minimal media are far more growth inhibited, and repression of $p t s G$ alone is not sufficient to rescue growth. In minimal media stress conditions, repression of $p t s G$ and activation of $y i g L$ are necessary, but not sufficient for full growth rescue (Sun and Vanderpool, 2013). These findings illustrate the poorly understood influence of nutrient availability on the severity of glucosephosphate stress. Moreover, these results highlight the fact that additional unknown SgrS targets are involved in the stress response.

Phosphosugar intermediates of central metabolism provide precursors for biomass and energy, yet, as illustrated by glucosephosphate stress, excessive accumulation of phosphosugars is detrimental to cell growth. Other types of phosphosugar stress also cause growth inhibition or cell lysis (Yarmolinsky et al., 1959; Englesberg et al., 1962; Irani and Maitra, 1977; Lee et al., 2009). In most cases, the mechanisms responsible for phosphosugarassociated inhibition or lysis have not been defined. However, recent work suggests that in some cases phosphosugars themselves are not directly inhibitory. Rather, accumulation of phosphosugars is accompanied by depletion of other metabolites, and stress is ameliorated by supplementation with the limiting metabolites (Lee et al., 2009, 2013; Richards et al., 2013). Glucose-phosphate stress is so far associated with accumulation of a few sugar-phosphate intermediates of upper glycolysis (Morita et al., 2003; Vanderpool and Gottesman, 2004; Sun and Vanderpool, 2013). A recent study implicates depletion of intermediates of lower glycolysis, particularly phosphoenolpyruvate
(PEP) as an important cause of glucose-phosphate stress. When $\alpha \mathrm{MG}$ is taken up and phosphorylated, it cannot be metabolized to replenish glycolytic intermediates. Thus, PEP utilized to drive $\alpha \mathrm{MG}$ uptake is not replaced via glycolytic metabolism. Under these conditions, SgrS regulation of target mRNAs and production of SgrT limits PEP consumption by reducing levels and activity of PtsG (Figure 1). In sgrS mutants, exposure to $\alpha \mathrm{MG}$ results in strong growth inhibition (Vanderpool and Gottesman, 2004; Richards et al., 2013) that is largely reversed by supplementing stressed cultures with glycolytic intermediates (Richards et al., 2013). The ratios of PEP to pyruvate seem to be particularly relevant for growth during glucose-phosphate stress. Increasing pyruvate levels during stress results in lysis of sgrS mutant cells, whereas increasing PEP levels rescues cell growth (Richards et al., 2013). The observation that stress (and growth inhibition) is more severe when cells are growing in minimal compared to rich media is also consistent with metabolite depletion as an underlying cause of glucose-phosphate stress. In rich media, cells do not have to synthesize many biosynthetic intermediates. In contrast, growth in minimal media requires de novo biosynthesis of amino acids. Thus, depletion of glycolytic intermediates during glucose-phosphate stress would have more severe effects on growth under conditions where these same intermediates are needed as precursors for biosynthesis. Consistent with this idea, supplementation of minimal media with amino acids improves stress recovery in minimal medium (Sun and Vanderpool, 2013).

The transcription factor SgrR also plays an important, but not fully characterized role in glucose-phosphate stress physiology. SgrR activates expression of $\operatorname{sgrS}$ and at least two other genes during glucose-phosphate stress: set $A$, encoding an efflux pump (Liu et al., 1999; Sun and Vanderpool, 2011), and alaC (formerly $y f d Z$ ), a glutamate-pyruvate aminotransferase (Vanderpool and Gottesman, 2007; Kim et al., 2010). The role of alaC in helping cells recover from glucose-phosphate stress is unknown. In contrast, set $A$, which is encoded just downstream of $s g r S$, is important for growth recovery under certain stress conditions (Sun and Vanderpool, 2011). Given its function as an efflux pump, the hypothesis that SetA was responsible for export of aMG was tested, but was not supported (Sun and Vanderpool, 2011). Thus, the role of SetA in glucose-phosphate stress also remains elusive.

\section{DISTRIBUTION AND FUNCTION OF SgrS IN $\gamma$-PROTEOBACTERIA IDENTIFICATION OF SgrS IN ENTERIC BACTERIA}

SgrS homologs were identified in many $\gamma$-Proteobacteria, including Escherichia sp., Salmonella sp., Shigella sp., Yersinia sp, Serratia sp., Klebsiella pneumoniae and Erwinia sp. (Horler and Vanderpool, 2009). The sgrR-sgrS intergenic region (containing the sgrS promoter) is highly conserved, suggesting that SgrR regulates $s g r S$ expression in all these organisms. All identified SgrS homologs contain a Rho-independent terminator and most possess an additional stem-loop structure upstream of the terminator; these two structures are important for Hfq binding to SgrS (Figure 2A) (Horler and Vanderpool, 2009; Otaka et al., 2011; Ishikawa et al., 2012). While the overall conservation of 
SgrS is low, a short stretch of $\sim 13$ nts near the SgrS $3^{\prime}$ end is nearly invariant (Figure 2B). This SgrS sequence is complementary to the translation initiation regions of $p t s G$ mRNAs in all species where an SgrS homolog was found (Horler and Vanderpool, 2009). Mutation of residues G176 and G178 within the conserved region of E. coli SgrS abrogates SgrS-mediated repression of $p t s G$ mRNA and prevents recovery from glucosephosphate stress (Maki et al., 2008). Introduction of analogous mutations in the conserved regions of SgrS homologs from Salmonella, E. carotovora, Y. pestis and K. pneumoniae similarly prevented regulation of $p t s G$ (Wadler and Vanderpool, 2009). Regulation of other targets is less well conserved among SgrS homologs. The SgrS sequences required for base pairing with manX are upstream of the conserved region (Figure 2C) and are poorly conserved among SgrS homologs. SgrS homologs from Salmonella and K. pneumoniae have the same predicted SgrS-man $X$ base pairing interaction and manX translation is regulated as expected. In contrast, E. carotovora and Y. pestis SgrS homologs have changes in the manX pairing site resulting in loss of complementarity to their cognate manX and were accordingly shown not to regulate manX translation (Rice and Vanderpool, 2011).

\section{CONSERVATION OF SgrT}

While the exact molecular function of SgrT has not been reported, available data strongly suggest that this small protein interacts directly with PtsG protein to inhibit its activity (Wadler and Vanderpool, 2007). Most SgrS homologs contain open reading frames similar in size to E. coli SgrT ( $\sim 40$ amino acids) (Horler and Vanderpool, 2009). While the primary amino acid sequence of putative SgrT homologs was not well conserved, homologs from Salmonella, Klebsiella, and Erwinia were functional when expressed in an E. coli sgrST mutant (Wadler and Vanderpool, 2009). Interestingly, some species with SgrS homologs appear to lack a functional SgrT. In Yersinia sp., SgrS appears to be truncated at the $5^{\prime}$ end, and SgrS from Yersinia species ranges in size from $\sim 85$ to $140 \mathrm{nt}$ and lacks the $s g r T$ open reading frame. In pathogenic E. coli O157:H7 strains, a point mutation in the SgrS $5^{\prime}$ region alters the $\operatorname{sgr} T$ start codon, presumably abrogating SgrT production in these strains.

Differential presence and absence of SgrT in organisms that possess SgrS led to a closer comparison of E. coli K12 and Salmonella SgrS sRNAs. In E. coli K12, sgrT alone (without the region of SgrS involved in base pairing with mRNAs) was not sufficient to allow growth rescue during glucose-phosphate stress conditions. This is in part due to very low levels of SgrT produced from the native sgrS allele in E. coli (Wadler and Vanderpool, 2009). E. coli SgrS has a sequence in the $5^{\prime}$ region that forms a structure that inhibits sgrT translation. On the other hand, Salmonella SgrS does not have the same inhibitory structure and therefore produces more SgrT than E. coli SgrS (Wadler and Vanderpool, 2009; Balasubramanian and Vanderpool, 2013). While native levels of SgrT production have not been investigated in Erwinia or Klebsiella species, it was observed that ectopic production of SgrT homologs from these organisms in an E. coli sgrST mutant rescued growth during glucose-phosphate stress (Wadler and Vanderpool, 2009). Thus, SgrT is functionally conserved when it is present, but levels of SgrT production vary among bacteria.

\section{SgrS REGULATION OF sOpD mRNA}

Although SgrS is conserved among enteric bacteria, divergence in primary sequence has resulted in species-specific target regulons, exemplified by the finding that Erwinia and Yersinia SgrS homologs do not regulate their cognate manXYZ homologs (Rice and Vanderpool, 2011). Another instance of species-specific regulation by SgrS is regulation of the Salmonella-specific gene sopD (Papenfort et al., 2012). SopD is an effector delivered to host cells through the Type 3 Secretion Systems (T3SSs) encoded on Salmonella pathogenicity island (SPI)-1 and SPI-2 (Brumell et al., 2003) and it functions as a general virulence factor in mice (Jiang et al., 2004; Bakowski et al., 2007). Regulation of sopD by SgrS involves base pairing interactions between the conserved region of SgrS and the early coding sequence of sopD mRNA (Figure 2C); the interaction inhibits translation initiation and stimulates sopD mRNA degradation (Papenfort et al., 2012). Interestingly, Salmonella encodes a second SopD protein, SopD2, which shares $42 \%$ identity with SopD and likely arose from a duplication (Jiang et al., 2004). The predicted SgrS-sopD2 base pairing interaction differs from SgrS-sopD at only a single position, a wobble G: $\mathrm{U}$ base pair instead of the G:C base pair. Remarkably, this interaction that differs by only a single hydrogen bond prevents regulation of sopD2 by SgrS (Papenfort et al., 2012).

While the biological significance of sopD regulation by SgrS is not yet clear, the inclusion of $\operatorname{sopD}$ in the Salmonella SgrS regulon illustrates plasticity in the evolution of sRNA regulons. The presence of sgrR-sgrS-sgrT in the same genomic context in pathogenic and non-pathogenic $\gamma$-proteobacteria (Horler and Vanderpool, 2009) suggests that this is an ancestral, or "core" RNA among these organisms. Yet, this core sRNA has acquired the ability to regulate a gene that was horizontally acquired by Salmonella. Studies of other SgrS homologs in pathogenic and non-pathogenic enteric bacteria will surely shed light on the breadth of regulatory activities of this fascinating dual-function sRNA.

\section{REFERENCES}

Bakowski, M. A., Cirulis, J. T., Brown, N. F., Finlay, B. B., and Brumell, J. H. (2007). SopD acts cooperatively with SopB during Salmonella enterica serovar Typhimurium invasion. Cell. Microbiol. 9, 2839-2855. doi: 10.1111/j.14625822.2007.01000.x

Balasubramanian, D., and Vanderpool, C. K. (2013). Deciphering the interplay between two independent functions of the small RNA regulator SgrS in Salmonella. J. Bacteriol. 195, 4620-4630. doi: 10.1128/JB.00586-13

Bandyra, K. J., Said, N., Pfeiffer, V., Gorna, M. W., Vogel, J., and Luisi, B. F. (2012). The seed region of a small RNA drives the controlled destruction of the target mRNA by the endoribonuclease RNase E. Mol. Cell 47, 943-953. doi: 10.1016/j.molcel.2012.07.015

Belasco, J. G. (2010). All things must pass: contrasts and commonalities in eukaryotic and bacterial mRNA decay. Nat. Rev. Mol. Cell Biol. 11, 467-478. doi: $10.1038 / \mathrm{nrm} 2917$

Beyer, D., Skripkin, E., Wadzack, J., and Nierhaus, K. H. (1994). How the ribosome moves along the mRNA during protein synthesis. J. Biol. Chem. 269, 30713-30717.

Bouvier, M., Sharma, C. M., Mika, F., Nierhaus, K. H., and Vogel, J. (2008). Small RNA binding to $5^{\prime}$ mRNA coding region inhibits translational initiation. Mol. Cell 32, 827-837. doi: 10.1016/j.molcel.2008.10.027 
Brumell, J. H., Kujat-Choy, S., Brown, N. F., Vallance, B. A., Knodler, L. A., and Finlay, B. B. (2003). SopD2 is a novel type III secreted effector of Salmonella typhimurium that targets late endocytic compartments upon delivery into host cells. Traffic 4, 36-48. doi: 10.1034/j.1600-0854.2003.40106.x

Carpousis, A. J. (2007). The RNA degradosome of Escherichia coli: an mRNAdegrading machine assembled on RNase E. Annu. Rev. Microbiol. 61, 71-87. doi: 10.1146/annurev.micro.61.080706.093440

Carpousis, A. J., Luisi, B. F., and McDowall, K. J. (2009). Endonucleolytic initiation of mRNA decay in Escherichia coli. Prog. Mol. Biol. Transl. Sci. 85, 91-135. doi: 10.1016/S0079-6603(08)00803-9

Darfeuille, F., Unoson, C., Vogel, J., and Wagner, E. G. (2007). An antisense RNA inhibits translation by competing with standby ribosomes. Mol. Cell 26, 381-392. doi: 10.1016/j.molcel.2007.04.003

Desnoyers, G., Bouchard, M. P., and Masse, E. (2013). New insights into small RNAdependent translational regulation in prokaryotes. Trends Genet. 29, 92-98. doi: 10.1016/j.tig.2012.10.004

Desnoyers, G., and Masse, E. (2012). Noncanonical repression of translation initiation through small RNA recruitment of the RNA chaperone Hfq. Genes Dev. 26, 726-739. doi: 10.1101/gad.182493.111

Desnoyers, G., Morissette, A., Prevost, K., and Masse, E. (2009). Small RNAinduced differential degradation of the polycistronic mRNA iscRSUA. EMBO J. 28, 1551-1561. doi: 10.1038/emboj.2009.116

Deutscher, J., Francke, C., and Postma, P. W. (2006). How phosphotransferase system-related protein phosphorylation regulates carbohydrate metabolism in bacteria. Microbiol. Mol. Biol. Rev. 70, 939-1031. doi: 10.1128/MMBR.00024-06

Dreyfus, M. (2009). Killer and protective ribosomes. Prog. Mol. Biol. Transl. Sci. 85, 423-466. doi: 10.1016/S0079-6603(08)00811-8

Englesberg, E., Anderson, R. L., Weinberg, R., Lee, N., Hoffee, P., Huttenhauer, G., et al. (1962). L-Arabinose-sensitive, L-ribulose 5-phosphate 4-epimerasedeficient mutants of Escherichia coli. J. Bacteriol. 84, 137-146.

Fender, A., Elf, J., Hampel, K., Zimmermann, B., and Wagner, E. G. (2010). RNAs actively cycle on the Sm-like protein Hfq. Genes Dev. 24, 2621-2626. doi: 10.1101/gad.591310

Frohlich, K. S., Papenfort, K., Fekete, A., and Vogel, J. (2013). A small RNA activates CFA synthase by isoform-specific mRNA stabilization. EMBO J. 32, 2963-2979. doi: 10.1038/emboj.2013.222

Gorke, B., and Stulke, J. (2008). Carbon catabolite repression in bacteria: many ways to make the most out of nutrients. Nat. Rev. Microbiol. 6, 613-624. doi: $10.1038 /$ nrmicro 1932

Haguenauer, R., and Kepes, A. (1972). NaF inhibition of phosphorylation and dephosphorylation involved in -methyl-D glucoside transport in E. coli K 12 . A $\mathrm{pH}$ dependent phenomenon sensitive to uncoupling agents. Biochimie 54, 505-512. doi: 10.1016/S0300-9084(72)80235-9

Holmqvist, E., Reimegard, J., Sterk, M., Grantcharova, N., Romling, U., and Wagner, E. G. (2010). Two antisense RNAs target the transcriptional regulator CsgD to inhibit curli synthesis. EMBO J. 29, 1840-1850. doi: 10.1038/emboj.2010.73

Hopkins, J. F., Panja, S., and Woodson, S. A. (2011). Rapid binding and release of Hfq from ternary complexes during RNA annealing. Nucleic Acids Res. 39, 5193-5202. doi: 10.1093/nar/gkr062

Horler, R. S., and Vanderpool, C. K. (2009). Homologs of the small RNA SgrS are broadly distributed in enteric bacteria but have diverged in size and sequence. Nucleic Acids Res. 37, 5465-5476. doi: 10.1093/nar/gkp501

Huttenhofer, A., and Noller, H. F. (1994). Footprinting mRNA-ribosome complexes with chemical probes. EMBO J. 13, 3892-3901.

Irani, M. H., and Maitra, P. K. (1977). Properties of Escherichia coli mutants deficient in enzymes of glycolysis. J. Bacteriol. 132, 398-410.

Ishikawa, H., Otaka, H., Maki, K., Morita, T., and Aiba, H. (2012). The functional Hfq-binding module of bacterial sRNAs consists of a double or single hairpin preceded by a U-rich sequence and followed by a $3^{\prime}$ poly(U) tail. RNA 18 , 1062-1074. doi: 10.1261/rna.031575.111

Jiang, X., Rossanese, O. W., Brown, N. F., Kujat-Choy, S., Galan, J. E., Finlay, B. B., et al. (2004). The related effector proteins SopD and SopD2 from Salmonella enterica serovar Typhimurium contribute to virulence during systemic infection of mice. Mol. Microbiol. 54, 1186-1198. doi: 10.1111/j.1365-2958.2004.04344.x

Kawamoto, H., Koide, Y., Morita, T., and Aiba, H. (2006). Base-pairing requirement for RNA silencing by a bacterial small RNA and acceleration of duplex formation by Hfq. Mol. Microbiol. 61, 1013-1022. doi: 10.1111/j.13652958.2006.05288.x
Kim, S. H., Schneider, B. L., and Reitzer, L. (2010). Genetics and regulation of the major enzymes of alanine synthesis in Escherichia coli. J. Bacteriol. 192, 5304-5311. doi: 10.1128/JB.00738-10

Koonin, E. V., and Tatusov, R. L. (1994). Computer analysis of bacterial haloacid dehalogenases defines a large superfamily of hydrolases with diverse specificity. Application of an iterative approach to database search. J. Mol. Biol. 244, 125-132. doi: 10.1006/jmbi.1994.1711

Lease, R. A., Cusick, M. E., and Belfort, M. (1998). Riboregulation in Escherichia coli: DsrA RNA acts by RNA:RNA interactions at multiple loci. Proc. Natl. Acad. Sci. U.S.A. 95, 12456-12461. doi: 10.1073/pnas.95.21.12456

Lee, S. J., Trostel, A., and Adhya, S. (2013). Metabolite changes signal genetic regulatory mechanisms for robust cell behavior. MBio. 5, e00972-e00913. doi: 10.1128/mBio.00972-13

Lee, S. J., Trostel, A., Le, P., Harinarayanan, R., Fitzgerald, P. C., and Adhya, S. (2009). Cellular stress created by intermediary metabolite imbalances. Proc. Natl. Acad. Sci. U.S.A. 106, 19515-19520. doi: 10.1073/pnas.0910586106

Link, T. M., Valentin-Hansen, P., and Brennan, R. G. (2009). Structure of Escherichia coli $\mathrm{Hfq}$ bound to polyriboadenylate RNA. Proc. Natl. Acad. Sci. U.S.A. 106, 19292-19297. doi: 10.1073/pnas.0908744106

Liu, J. Y., Miller, P. F., Willard, J., and Olson, E. R. (1999). Functional and biochemical characterization of Escherichia coli sugar efflux transporters. J. Biol. Chem. 274, 22977-22984. doi: 10.1074/jbc.274.33.22977

Maki, K., Morita, T., Otaka, H., and Aiba, H. (2010). A minimal base-pairing region of a bacterial small RNA SgrS required for translational repression of ptsG mRNA. Mol. Microbiol. 76, 782-792. doi: 10.1111/j.1365-2958.2010.07141.x

Maki, K., Uno, K., Morita, T., and Aiba, H. (2008). RNA, but not protein partners, is directly responsible for translational silencing by a bacterial Hfqbinding small RNA. Proc. Natl. Acad. Sci. U.S.A. 105, 10332-10337. doi: 10.1073/pnas.0803106105

Masse, E., Escorcia, F. E., and Gottesman, S. (2003). Coupled degradation of a small regulatory RNA and its mRNA targets in Escherichia coli. Genes Dev. 17, 2374-2383. doi: 10.1101/gad.1127103

Masse, E., and Gottesman, S. (2002). A small RNA regulates the expression of genes involved in iron metabolism in Escherichia coli. Proc. Natl. Acad. Sci. U.S.A. 99, 4620-4625. doi: 10.1073/pnas.032066599

Mikulecky, P. J., Kaw, M. K., Brescia, C. C., Takach, J. C., Sledjeski, D. D., and Feig, A. L. (2004). Escherichia coli Hfq has distinct interaction surfaces for DsrA, rpoS and poly(A) RNAs. Nat. Struct. Mol. Biol. 11, 1206-1214. doi: 10.1038/nsmb858

Moller, T., Franch, T., Udesen, C., Gerdes, K., and Valentin-Hansen, P. (2002). Spot 42 RNA mediates discoordinate expression of the E. coli galactose operon. Genes Dev. 16, 1696-1706. doi: 10.1101/gad.231702

Morfeldt, E., Taylor, D., Von Gabain, A., and Arvidson, S. (1995). Activation of alpha-toxin translation in Staphylococcus aureus by the trans-encoded antisense RNA, RNAIII. EMBO J. 14, 4569-4577.

Morita, T., El-Kazzaz, W., Tanaka, Y., Inada, T., and Aiba, H. (2003). Accumulation of glucose 6-phosphate or fructose 6-phosphate is responsible for destabilization of glucose transporter mRNA in Escherichia coli. J. Biol. Chem. 278, 15608-15614. doi: 10.1074/jbc.M300177200

Morita, T., Maki, K., and Aiba, H. (2005). RNase E-based ribonucleoprotein complexes: mechanical basis of mRNA destabilization mediated by bacterial noncoding RNAs. Genes Dev. 19, 2176-2186. doi: 10.1101/gad.1330405

Morita, T., Mochizuki, Y., and Aiba, H. (2006). Translational repression is sufficient for gene silencing by bacterial small noncoding RNAs in the absence of mRNA destruction. Proc. Natl. Acad. Sci. U.S.A. 103, 4858-4863. doi: 10.1073/pnas.0509638103

Obana, N., Shirahama, Y., Abe, K., and Nakamura, K. (2010). Stabilization of Clostridium perfringens collagenase mRNA by VR-RNA-dependent cleavage in $5^{\prime}$ leader sequence. Mol. Microbiol. 77, 1416-1428. doi: 10.1111/j.13652958.2010.07258.x

Otaka, H., Ishikawa, H., Morita, T., and Aiba, H. (2011). PolyU tail of rhoindependent terminator of bacterial small RNAs is essential for Hfq action. Proc. Natl. Acad. Sci. U.S.A. 108, 13059-13064. doi: 10.1073/pnas.1107050108

Papenfort, K., Podkaminski, D., Hinton, J. C., and Vogel, J. (2012). The ancestral SgrS RNA discriminates horizontally acquired Salmonella mRNAs through a single G-U wobble pair. Proc. Natl. Acad. Sci. U.S.A. 109, E757-764. doi: 10.1073/pnas.1119414109

Papenfort, K., Sun, Y., Miyakoshi, M., Vanderpool, C. K., and Vogel, J. (2013). Small RNA-mediated activation of sugar phosphatase mRNA regulates glucose homeostasis. Cell 153, 426-437. doi: 10.1016/j.cell.2013.03.003 
Pfeiffer, V., Papenfort, K., Lucchini, S., Hinton, J., and Vogel, J. R. (2009). Coding sequence targeting by MicC RNA reveals bacterial mRNA silencing downstream of translational initiation. Nat. Struct. Mol. Biol. 16, 840-846. doi: 10.1038/nsmb.1631

Postma, P. W., Lengeler, J. W., and Jacobson, G. R. (1993). Phosphoenolpyruvate: carbohydrate phosphotransferase systems of bacteria. Microbiol. Rev. 57, 543-594.

Prevost, K., Desnoyers, G., Jacques, J. F., Lavoie, F., and Masse, E. (2011). Small RNA-induced mRNA degradation achieved through both translation block and activated cleavage. Genes Dev. 25, 385-396. doi: 10.1101/gad.2001711

Prevost, K., Salvail, H., Desnoyers, G., Jacques, J. F., Phaneuf, E., and Masse, E. (2007). The small RNA RyhB activates the translation of shiA mRNA encoding a permease of shikimate, a compound involved in siderophore synthesis. Mol. Microbiol. 64, 1260-1273. doi: 10.1111/j.1365-2958.2007.05733.x

Rice, J. B., Balasubramanian, D., and Vanderpool, C. K. (2012). Small RNA binding-site multiplicity involved in translational regulation of a polycistronic mRNA. Proc. Natl. Acad. Sci. U.S.A. 109, E2691-2698. doi: 10.1073/pnas.12079 27109

Rice, J. B., and Vanderpool, C. K. (2011). The small RNA SgrS controls sugarphosphate accumulation by regulating multiple PTS genes. Nucleic Acids Res. 39, 3806-3819. doi: 10.1093/nar/gkq1219

Richards, G. R., Patel, M. V., Lloyd, C. R., and Vanderpool, C. K. (2013). Depletion of glycolytic intermediates plays a key role in glucose-phosphate stress in Escherichia coli. J. Bacteriol. 195, 4816-4825. doi: 10.1128/JB.00705-13

Salim, N. N., Faner, M. A., Philip, J. A., and Feig, A. L. (2012). Requirement of upstream Hfq-binding (ARN)x elements in glmS and the Hfq C-terminal region for GlmS upregulation by sRNAs GlmZ and GlmY. Nucleic Acids Res. 40, 8021-8032. doi: 10.1093/nar/gks392.

Salim, N. N., and Feig, A. L. (2010). An upstream Hfq binding site in the fhlA mRNA leader region facilitates the OxyS-fhlA interaction. PLoS ONE 5:e13028. doi: 10.1371/journal.pone.0013028.

Sharma, C. M., Darfeuille, F., Plantinga, T. H., and Vogel, J. (2007). A small RNA regulates multiple $\mathrm{ABC}$ transporter mRNAs by targeting C/A-rich elements inside and upstream of ribosome-binding sites. Genes Dev. 21, 2804-2817. doi: 10.1101/gad.447207

Sledjeski, D. D., Whitman, C., and Zhang, A. (2001). Hfq is necessary for regulation by the untranslated RNA DsrA. J. Bacteriol. 183, 1997-2005. doi: 10.1128/JB.183.6.1997-2005.2001

Soper, T. J., and Woodson, S. A. (2008). The rpoS mRNA leader recruits Hfq to facilitate annealing with DsrA sRNA. RNA. 14, 1907-1917. doi: 10.1261/rna.1110608.

Soper, T., Mandin, P., Majdalani, N., Gottesman, S., and Woodson, S. A. (2010). Positive regulation by small RNAs and the role of Hfq. Proc. Natl. Acad. Sci. U.S.A. 107, 9602-9607. doi: 10.1073/pnas.1004435107

Sun, Y., and Vanderpool, C. K. (2011). Regulation and function of Escherichia coli sugar efflux transporter A (SetA) during glucose-phosphate stress. J. Bacteriol. 193, 143-153. doi: 10.1128/JB.01008-10

Sun, Y., and Vanderpool, C. K. (2013). Physiological consequences of multipletarget regulation by the small RNA SgrS in Escherichia coli. J. Bacteriol. 195, 4804-4815. doi: 10.1128/JB.00722-13
Valentin-Hansen, P., Eriksen, M., and Udesen, C. (2004). The bacterial Sm-like protein Hfq: a key player in RNA transactions. Mol. Microbiol. 51, 1525-1533. doi: 10.1111/j.1365-2958.2003.03935.x

Vanderpool, C. K., and Gottesman, S. (2004). Involvement of a novel transcriptional activator and small RNA in post-transcriptional regulation of the glucose phosphoenolpyruvate phosphotransferase system. Mol. Microbiol. 54, 1076-1089. doi: 10.1111/j.1365-2958.2004.04348.x

Vanderpool, C. K., and Gottesman, S. (2007). The novel transcription factor SgrR coordinates the response to glucose-phosphate stress. J. Bacteriol. 189, 2238-2248. doi: 10.1128/JB.01689-06

Wadler, C. S., and Vanderpool, C. K. (2007). A dual function for a bacterial small RNA: SgrS performs base pairing-dependent regulation and encodes a functional polypeptide. Proc. Natl. Acad. Sci. U.S.A. 104, 20454-20459. doi: 10.1073/pnas.0708102104

Wadler, C. S., and Vanderpool, C. K. (2009). Characterization of homologs of the small RNA SgrS reveals diversity in function. Nucleic Acids Res. 37, 5477-5485. doi: 10.1093/nar/gkp591

Winkler, H. H. (1971). Efflux and the steady state in alpha-methylglucoside transport in Escherichia coli. J. Bacteriol. 106, 362-368.

Yarmolinsky, M. B., Wiesmeyer, H., Kalckar, H. M., and Jordan, E. (1959). Hereditary defects in galactose metabolism in Escherichia Coli mutants, Ii. Galactose-induced sensitivity. Proc. Natl. Acad. Sci. U.S.A. 45, 1786-1791. doi: 10.1073/pnas.45.12.1786

Zhang, A., Schu, D. J., Tjaden, B. C., Storz, G., and Gottesman, S. (2013). Mutations in interaction surfaces differentially impact E. coli Hfq association with small RNAs and their mRNA targets. J. Mol. Biol. 425, 3678-3697. doi: 10.1016/j.jmb.2013.01.006

Zhang, A., Wassarman, K. M., Ortega, J., Steven, A. C., and Storz, G. (2002). The Sm-like Hfq protein increases OxyS RNA interaction with target mRNAs. Mol. Cell 9, 11-22. doi: 10.1016/S1097-2765(01)00437-3

Zhang, A., Wassarman, K. M., Rosenow, C., Tjaden, B. C., Storz, G., and Gottesman, S. (2003). Global analysis of small RNA and mRNA targets of Hfq Mol. Microbiol. 50, 1111-1124. doi: 10.1046/j.1365-2958.2003.03734.x

Conflict of Interest Statement: The authors declare that the research was conducted in the absence of any commercial or financial relationships that could be construed as a potential conflict of interest.

Received: 05 March 2014; accepted: 22 April 2014; published online: 08 May 2014. Citation: Bobrovskyy $M$ and Vanderpool CK (2014) The small RNA SgrS: roles in metabolism and pathogenesis of enteric bacteria. Front. Cell. Infect. Microbiol. 4:61. doi: 10.3389/fcimb.2014.00061

This article was submitted to the journal Frontiers in Cellular and Infection Microbiology.

Copyright (c) 2014 Bobrovskyy and Vanderpool. This is an open-access article distributed under the terms of the Creative Commons Attribution License (CC BY). The use, distribution or reproduction in other forums is permitted, provided the original author(s) or licensor are credited and that the original publication in this journal is cited, in accordance with accepted academic practice. No use, distribution or reproduction is permitted which does not comply with these terms. 